\title{
Identification of key tumorigenesis-related genes and their microRNAs in colon cancer
}

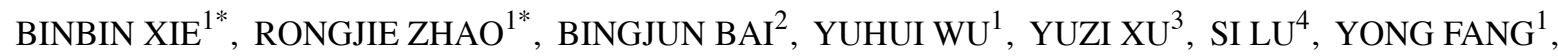 \\ ZHANGGUI WANG ${ }^{5}$, EWETSE PAUL MASWIKITI ${ }^{3}$, XIAOYUN ZHOU ${ }^{6}$, \\ HONGMING PAN ${ }^{1,6}$ and WEIDONG HAN ${ }^{1}$
}

\begin{abstract}
Departments of ${ }^{1}$ Medical Oncology, ${ }^{2}$ Colorectal Surgery, Sir Run Run Shaw Hospital, School of Medicine, Zhejiang University, Hangzhou, Zhejiang 310016; ${ }^{3}$ Department of Sports Medicine, School of Medicine, Zhejiang University, Hangzhou, Zhejiang 310012; ${ }^{4}$ Department of Oncological Surgery, Hangzhou First People's Hospital, The Fourth Clinical Medical College Affiliated to Zhejiang Chinese Medical University, Hangzhou, Zhejiang 310002;

${ }^{5}$ Department of Radiotherapy, The Second People's Hospital of Anhui Province, Hefei, Anhui 230041;

${ }^{6}$ Department of Medical Oncology, Xiasha Campus, Sir Run Run Shaw Hospital, College of Medicine, Zhejiang University, Hangzhou, Zhejiang 310016, P.R. China
\end{abstract}

Received April 23, 2018; Accepted September 13, 2018

DOI: $10.3892 / o r .2018 .6726$

\begin{abstract}
Colorectal cancer (CRC) is one of the most common malignant tumors and its development involves multi-gene driven processes that affect the digestive system. The objective of this study was to identify tumorigenesis-associated gene signatures using microarray expression profiling data. The gene expression profiling of GSE39582, a dataset containing 566 colon cancer samples and 19 non-tumoral colorectal mucosae was downloaded from Gene Expression Omnibus (GEO) database. A total of 439 differentially expressed genes (DEGs) were extracted by GEO2R. Many of these DEGs were cancer-related, involved in the regulation of cell proliferation, extracellular matrix (ECM)-receptor interaction and phosphatidylinositol 3-kinase (PI3K)-Akt signaling pathway according to the results of pathway enrichment analysis in Database for Annotation, Visualization and Integrated Discovery (DAVID). Then, 10 genes were predicted to play an important role in the development of CRC. Transient receptor potential cation channel, subfamily $\mathrm{M}$, and member 6 (TRPM6), a member of 10 hub genes, was confirmed to be downregulated in $16(80 \%)$ of 20 colon cancer tissues using quantitative polymerase chain reaction (qPCR) technology. Furthermore, high expression of TRPM6 was indicative of
\end{abstract}

Correspondence to: Dr Weidong Han or Dr Hongming Pan, Department of Medical Oncology, Sir Run Run Shaw Hospital, School of Medicine, Zhejiang University, 3 East Qinchun Road, Hangzhou, Zhejiang 310016, P.R. China

E-mail: hanwd@zju.edu.cn; panhongming@zju.edu.cn

${ }^{*}$ Contributed equally

Key words: colorectal cancer, tumorigenesis, TRPM6, microRNAs a prolonged overall survival (OS) in CRC patients through the analysis of GSE39582. Hsa-let-7g and hsa-let-7f-1 were believed to be the regulatory miRNAs of TRPM6 by TCGA and miRanda database. In conclusion, this study may play a critical role in promoting the discovery of potential targets for diagnosis, treatment and prognosis of CRC.

\section{Introduction}

Colorectal cancer (CRC) is one of the most commonly diagnosed malignant tumors and the primary cause of cancer-related deaths worldwide. In 2017, >135,000 individuals with newly diagnosed CRC and over 50,000 deaths from the disease were reported in the United States. In spite of 58\% new cases of patients over 65 year of age, the number of patients under the age of 65 diagnosed with CRC is exponentially increasing $(1,2)$. CRC is caused by many factors, such as genetics, lifestyle and environment (3). In addition, there is solid evidence that tumorigenesis of CRC is a multi-gene and multi-pathway driven process (4-6). More than $70 \%$ of colorectal adenoma occur in the APC gene mutation site at an early stage and further promotes adenocarcinoma by activating mutations of the KRAS gene and inactivating TP53 gene. The mutations in these representative genes are often accompanied by changes in the number and structure of chromosomes. More than 15\% of sporadic CRCs occur by other ways, including the $\mathrm{CpG}$ island methylator phenotype, activation of BRAF oncogene mutations and MLH1 gene promoter methylation (7). Several signaling pathways such as Wnt-associated signaling pathway, Hippo pathway and PI3K pathway have been associated with CRC (8-10). Many reports have indicated the abnormal expression of certain miRNAs, which also induce CRC cell proliferation and migration (11). To date, the fundamental pathophysiology of the disease has not been fully elucidated, and remains a major obstacle for clinical diagnosis and treatment. 
In the last decade, microarray technology has been broadly used to filter out CRC-specific differentially expressed genes (DEGs). Iwaya et al identified DEGs between CRC patients and normal colon epithelium (12). Yan et al also identified potential biomarkers for the prognosis and prevention of CRC (13). Bioinformatic methods owing to their efficiency in dealing with high-throughput data are currently now in use, but the most representative DEGs or pathways still need to be identified. In addition, bioinformatic study combining mRNA and miRNAs to investigate the pathophysiological mechanisms of CRC has seldom been put forward. Thus, our research aimed to associate mRNA with microRNAs (miRNAs) to promote the discovery of potential diagnostic and therapeutic targets.

In the present study, we utilized the expression profiling data submitted by Marisa et al (14) to identify the DEGs between colon cancer samples and normal mucosa in Gene Expression Omnibus (GEO) 2R (https://www.ncbi. nlm.nih.gov/geo/geo2r/). Combining with the data of The Cancer Genome Atlas (TCGA) (https://portal.gdc.cancer. gov/), Oncomine (https://www.oncomine.org/), the tools of Database for Annotation, Visualization and Integrated Discovery (DAVID), Search Tool for the Retrieval of Interacting Genes (STRING) (https://string-db.org/), Cytoscape (http://www.cytoscape.org/), quantitative polymerase chain reaction (qPCR) and western blotting, the hub genes and its regulatory microRNAs (miRNAs) were screened and explored further.

\section{Materials and methods}

Identification of DEGs. Gene expression profiling of GSE39582 (14), a dataset based on Agilent GPL570 platform (Affymetrix Human Genome U133 Plus 2.0 Array), was downloaded from the GEO (https://www.ncbi.nlm.nih. gov/geo/) database. It contained 585 samples, and was divided into 2 groups: the tumor group consisted of 566 colon cancers, while the normal group consisted of 19 non-tumoral colorectal mucosa in GEO2R, an R-based web application (15). The genes that met the conditions of $\log 2$ fold change $(\log \mathrm{FC})$ of $\geq 2$ and P-value $<0.05$ were considered as DEGs. Then we applied hierarchical clustering analysis to classify the data in Morpheus (https://software.broadinstitute.org/morpheus/).

Enrichment analysis of DEGs. Gene Ontology (GO) database (http://www.geneontology.org/) described the facilities of genes and their products from three distinct biologic aspects: Biological process (BP), cellular component (CC) and molecular function (MF) (16). Kyoto Encyclopedia of Genes and Genomes pathway (KEGG) (http://www.kegg. jp), a comprehensive knowledge database, plays an important role in both functional interpretation and practical application of genomic information (17). The DEG list was uploaded to DAVID (v 6.8) to obtain enriched GO terms and significant pathway analysis with $\mathrm{P}<0.05$.

Assessment of PPI network. STRING database is an online database of known and predicted protein-protein interactions (PPIs). STRING (version 10.0) includes $9,643,763$ proteins from 2,031 living organisms. To assess the interactions among DEGs, we mapped them to STRING database and the coactions with a combined score of $>0.4$ were considered. Then, the PPI networks were visualized using Cytoscape software and the modules of DEGs were established by the Molecular Complex Detection (MCODE) with the concrete selection standards, which were as follows: MCODE scores $>2$ and number of nodes $>9$. In addition, hub genes were exported. Moreover, the KEGG pathway enrichment analysis was performed for genes within these modules separately.

Analysis of expression level of hub gene in TCGA and Oncomine. To obtain a picture of the hub genes' quality for further research, we compared the corresponding expression information using TCGA and Oncomine database. The gene expression quantification data of colorectal adenocarcinoma were downloaded from the TCGA database and it consisted of 521 individual data files, metadata and cart. Then, we obtained the list of DEGs between 480 colorectal adenocarcinoma and 41 normal samples using edgeR package (http://www. bioconductor.org/packages/release/bioc/html/edgeR.html) in $\mathrm{R}$ software with the following criteria: $\log \mathrm{FC} \geq 1$ and $\mathrm{P}<0.05$. Moreover, we searched the expression profile of the hub genes in Oncomine database, respectively with the following conditions: Gene, Cancer vs. Normal Analysis and Colorectal cancer type.

Survival analysis of hub genes. On the basis of the survival prognosis information of colon cancer patients in GSE39582, we calculated the survival curves of hub genes using GraphPad Prism version 6.0 (GraphPad Software, Inc., La Jolla, CA, USA) and $\mathrm{P}<0.05$ was thought to be significant.

Patients and tissue samples. In terms of the conditions, where high expression of TRPM6 was linked with a better overall survival (OS), 20 non-selected CRC samples were applied to perform qPCR to validate the expression difference of TRPM6 in colon cancer and normal colon mucosa (10 cm away from visible tumor edges). These experimental samples were collected at the Sir Run Run Shaw Hospital of Zhejiang University between January 2004 and December 2006. There were 16 males and 4 females (average age, 66.4 years; range, $49-88$ years). The pathological stage was defined according to UICC/AJCC and TNM classification system (https://www.uicc.org/resources/tnm). The details are shown in Table I. Each experimental sample was divided into two parts, one was used for histopathological evaluation and one for total RNA extraction. Research was authorized by the Ethics Committee of Sir Run Run Shaw Hospital and informed consent was obtained from all participating patients. The reference number was 20180226-88.

qPCR validation of TRPM6. The RNA of tissue samples was extracted using Trizol reagent (Invitrogen; Thermo Fisher Scientific, Inc., Waltham, MA, USA) following the manufacturer's instructions. RNA was quantified by applying a NanoDrop 2000c spectrophotometer (Thermo Fisher Scientific, Inc., Waltham, MA, USA). cDNA was synthesized using an RNeasy Mini Kit (Takara, Kyoto, Japan). qPCR analysis was performed with SYBR Green Master Mix (Takara). qPCR was performed at $95^{\circ} \mathrm{C}$ for $5 \mathrm{~min}, 45$ cycles of $95^{\circ} \mathrm{C}$ for $5 \mathrm{sec}$ and $60^{\circ} \mathrm{C}$ for $30 \mathrm{sec} ; 1$ cycle of $95^{\circ} \mathrm{C}$ for $5 \mathrm{sec}$, 
Table I. Histopathological characteristics of the CRC patients.

\begin{tabular}{llcllll}
\hline Patient & Sex & $\begin{array}{c}\text { Age } \\
\text { (years) }\end{array}$ & \multicolumn{1}{c}{$\begin{array}{c}\text { Location } \\
\text { of tumor }\end{array}$} & $\begin{array}{c}\text { Differentiation } \\
\text { grade }\end{array}$ & $\begin{array}{c}\text { TNM } \\
\text { staging }\end{array}$ & $\begin{array}{c}\text { UICC } \\
\text { staging }\end{array}$ \\
\hline 1 & Male & 88 & Rectum & Well-moderate & T3N2M1 & IV \\
2 & Female & 64 & Rectum & Well & T3N1M0 & IIIB \\
3 & Female & 63 & Sigmoid colon & Moderate & T3N2M0 & IIIC \\
4 & Male & 87 & Ileocecal junction & Moderate & T3N0M0 & IIIA \\
5 & Female & 56 & Rectum & Moderate & T3N2M0 & IIIC \\
6 & Male & 49 & Rectum & Moderate & T2N0M0 & I \\
7 & Male & 79 & Rectum & Well & T1N0M0 & I \\
8 & Male & 62 & Rectum & Well & T3N0M0 & IIA \\
9 & Female & 54 & Sigmoid colon & Woderate & T3N1M0 & IIIB \\
10 & Male & 79 & Sigmoid colon & Poor & T3N0M0 & IIA \\
11 & Male & 61 & Sigmoid colon & Well-moderate & T4N0M0 & IIIC \\
12 & Male & 50 & Sigmoid colon & Moderate & T3N0M0 & IIA \\
13 & Male & 64 & Sigmoid colon & Moderate-poor & T3N1M0 & IIIB \\
14 & Male & 71 & Sigmoid colon & Well-moderate & T3N0M0 & IIA \\
15 & Male & 61 & Ascending colon & Well-moderate & T3N1M1 & IV \\
16 & Male & 84 & Ascending colon & Well-moderate & T3N1M1 & IV \\
17 & Male & 59 & Hepatic flexure of colon & T3N0M0 & IIA \\
18 & Male & 59 & Ascending colon & Well & T3N0M1 & IV \\
19 & Male & 80 & Ascending colon & Ascending colon & Moderate & \\
20 & Male & 57 & & & &
\end{tabular}

CRC, colorectal cancer.

$60^{\circ} \mathrm{C}$ for $1 \mathrm{~min}$ and $95^{\circ} \mathrm{C}$ for $15 \mathrm{sec}$ and finally, $50^{\circ} \mathrm{C}$ for $30 \mathrm{sec}$. Relative expression was analyzed using the $2^{-\Delta \Delta \mathrm{Ca}}$ method (18). Expression of mRNA was normalized according to $\beta$-actin. The primers used were as follows: $\beta$-actin_forward, ACTCTT CCAGCCTTCCTTCC and $\beta$-actin_reverse, CGTCATACTC CTGCTTGCTG; TRPM6_forward, TCCTGTCTGAT GATGGGACC and TRPM6_reverse, TCTTGAGCGGCAG TGTATTTTC. We designed the primers on online tools (https://www.genscript.com/tools/real-time-pcr-tagman-prim er-design-tool) and these were synthesized by Shanghai Generay Biotech Co. Ltd. (Shanghai, China). We validated the specificity of primers with colon tissue by PCR and then running agarose gel (data not shown).

Western blotting of TRPM6. Tissue samples were directly extracted with radioimmunoprecipitation assay (RIPA) lysis buffer combined with protease inhibitor cocktail to quantify protein expression levels of TRPM6 and GAPDH. The extracted proteins were prepared and resolved by $10 \%$ SDS-PAGE and then transferred onto PVDF membranes (Millipore, Billerica, MA, USA). Membranes were blocked by using 5\% dry milk dissolved in TBST. The membranes were then incubated overnight at $4^{\circ} \mathrm{C}$ with antibodies against TRPM6 (BBI Life Sciences Corp., Shanghai, China; 1:1,000 dilution; cat. no. D162419) and GAPDH (Cell Signaling Technology, Inc., Danvers, MA, USA; 1:1,000 dilution; cat. no. 5174). After washing in Tris-buffered saline with Tween (TBST), the membranes were incubated for $2 \mathrm{~h}$ in
HRP-conjugated secondary antibodies (BBI Life Sciences Corp.; 1:1,0000 dilution; cat. no. D110058-0100,) at room temperature. Excess secondary antibodies (BBI Life Sciences Corp.; 1:1,0000 dilution; cat. no. D110058-0100) were washed and rinsed off from the membranes with TBST. Signals were visualized with an enhanced chemiluminescence kit (Biological Industries, Kibbutz Beth HaEmek, Israel). GAPDH was used as a loading control.

Identification of differentially expressed miRNAs (DEMs). Similar to the extraction of DEGs from TCGA database, the miRNA expression quantification data of colorectal adenocarcinoma was downloaded and it consisted of 465 individual data files, metadata and cart. Then, we obtained some results of DEMs between 457 colorectal adenocarcinoma and 8 normal samples using edgeR package with the qualification of $\log \mathrm{FC} \geq 1$ and $\mathrm{P}$-value $<0.05$.

Prediction of regulatory miRNAs of TRPM6. To locate the regulatory miRNAs of TRPM6 in CRC, we downloaded the files of Human Good mirSVR score, Conserved miRNA and Good mirSVR score, Non-conserved miRNAs from miRanda database (http://www.microrna.org/) to forecast the relationship between TRPM6 and miRNAs. The results of the TCGA-DEMs demonstrated intersection elements via the VennDiagram package (https://cran.r-project. org/web/packages/VennDiagram/index.html) in R software eventually. 


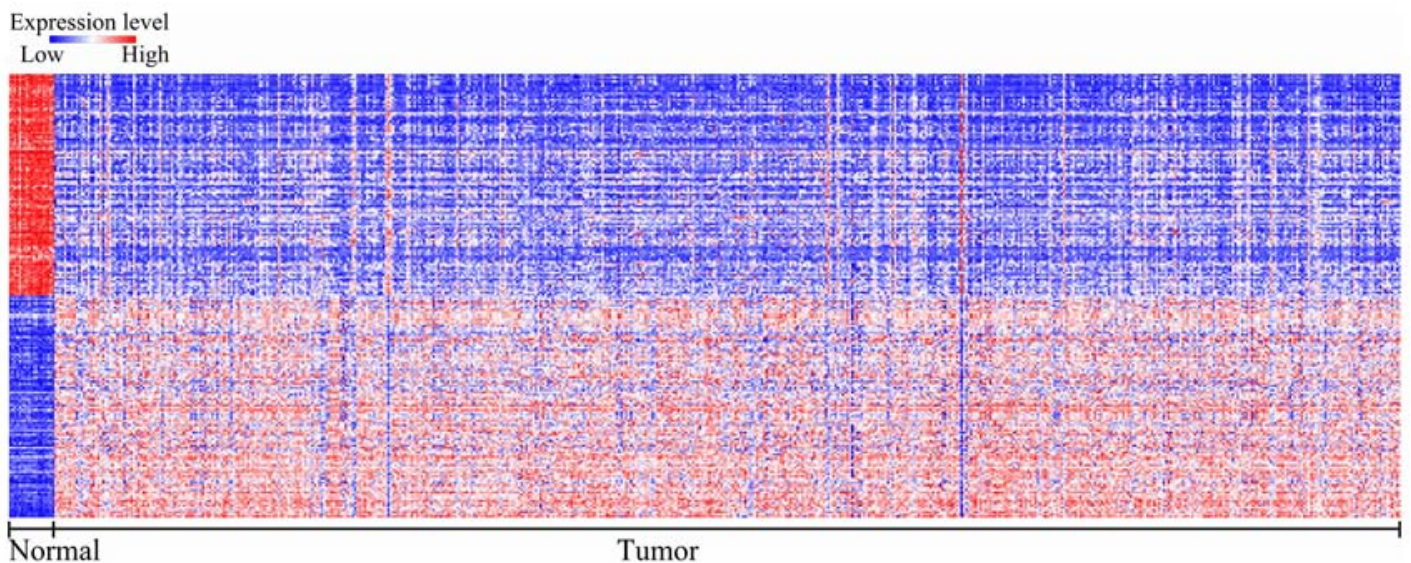

Figure 1. Heatmap of the top 100 differentially expressed genes (DEGs) in colon cancer and normal colon mucosa. A total of 439 DEGs were identified in GSE39582 between the two groups using Gene Expression Omnibus (GEO) $2 R$ with the following criteria: $P<0.05$ and $\log 2$ fold change (logFC) $\geq 2$. The expression of 50 upregulated genes and 50 downregulated genes was imported into Morpheus, an online heat map-making tool. Coloring illustrates the high expression (red) and low expression (blue) of genes.

A

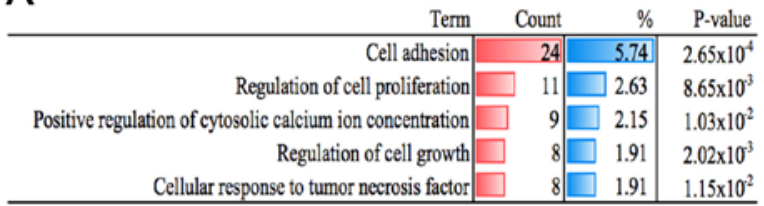

C

\begin{tabular}{|c|c|c|c|}
\hline Term & Count & $\%$ & P-value \\
\hline Extracellular matrix structural constituent & 11 & 2.63 & $1.85 \times 10^{6}$ \\
\hline Metalloendopeptidase activity & 13 & 3.11 & $6.96 \times 10^{-6}$ \\
\hline CXCR chemokine receptor binding & 5 & $\square 1.20$ & $2.62 \times 10^{5}$ \\
\hline Chloride channel activity & 8 & 1.91 & $1.68 \times 10^{4}$ \\
\hline Carbonate dehydratase activity & 5 & $\square 1.20$ & $1.91 \times 10^{4}$ \\
\hline
\end{tabular}

B

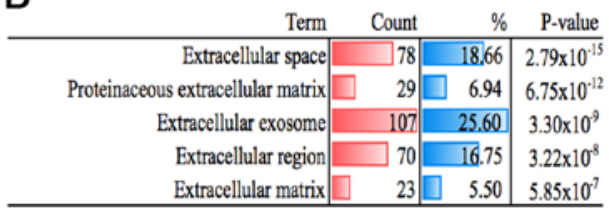

D

\begin{tabular}{|c|c|c|c|}
\hline Term & Count & $\%$ & P-value \\
\hline ECM-receptor interaction & 710 & 2.4 & $3.87 \times 10^{4}$ \\
\hline Chemical carcinogenesis & 8 & 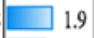 & $4.50 \times 10^{2}$ \\
\hline PPAR signaling pathway & 7 & $\square 1.7$ & $7.50 \times 10^{-3}$ \\
\hline Drug metabolism - cytochrome P450 & 7 & 1.7 & $8.05 \times 10^{3}$ \\
\hline PI3K-Akt signaling pathway & 15 & 3.6 & $6.31 \times 10^{-3}$ \\
\hline
\end{tabular}

Figure 2. Gene Ontology (GO) and Kyoto Encyclopedia of Genes and Genomes pathway (KEGG) enrichment analyses of DEGs. The list of DEGs was uploaded to the Database for Annotation, Visualization and Integrated Discovery (DAVID). Then, GO was performed from three different aspects: (A) biological process (BP), (B) cellular component (CC) and (C) molecular function (MF). (D) Likewise, KEGG was also performed to ensure the correlation between DEGs and tumorigenesis of colon cancer. Moreover, only the results with $\mathrm{P}<0.05$ were considered to be significant.

Statistical analysis. qPCR results are presented by the use of Graph Pad Prism (version 6.0; GraphPad Software, Inc., La Jolla, CA, USA). Data are presented as the mean \pm standard error of the mean. Independent samples t-test was performed for data comparison. $\mathrm{P}<0.05$ was considered to indicate a statistically significant difference.

\section{Results}

An overview of the expression levels of the DEGs. To identify the gene signatures during the development of $\mathrm{CRC}$, we compared the expression profiling of the array between colon cancer and non-tumoral mucosae using GEO2R. A total of 439 DEGs were found to play a role in carcinogenesis with $\mathrm{P}<0.05$ and $\log \mathrm{FC} \geq 2.0$ criteria, and top 50 genes were upregulated and downregulated, which was shown in heat map format performed in Morpheus (https://software.broadinstitute.org/morpheus/) (Fig. 1). These results indicated that colon cancer carcinogenesis is a complex multi-factorial process, which involves several genes.
DEGs are involved in CRC-associated molecular processes. To further investigate the potential mechanisms, we uploaded all the DEGs to DAVID, and then GO functional annotation and KEGG pathway enrichment analyses were conducted separately. As shown in Fig. 2A, many biological processes, such as cellular response to tumor necrosis factor (TNF), regulation of cell proliferation, positive regulation of cytosolic calcium ion concentration, cell adhesion and regulation of cell growth were identified. Additionally, the results of $\mathrm{CC}$ and MF are displayed in Fig. 2B and C. As for the KEGG results, DEGs were found to be involved in pathways including chemical carcinogenesis, ECM-receptor interaction, phosphatidylinositol 3-kinase (PI3K)-Akt signaling pathway, chemical carcinogenesis, peroxisome proliferator-activated receptor (PPAR) signaling pathway and drug metabolism-cytochrome P450 (Fig. 2D). These results showed that the DEGs were closely related to cancer and warranted identification.

Key modules and genes were screened out from the PPI network. The PPI network was constructed by the Search Tool for the Retrieval of Interacting Genes (STRING, version 10.0). 


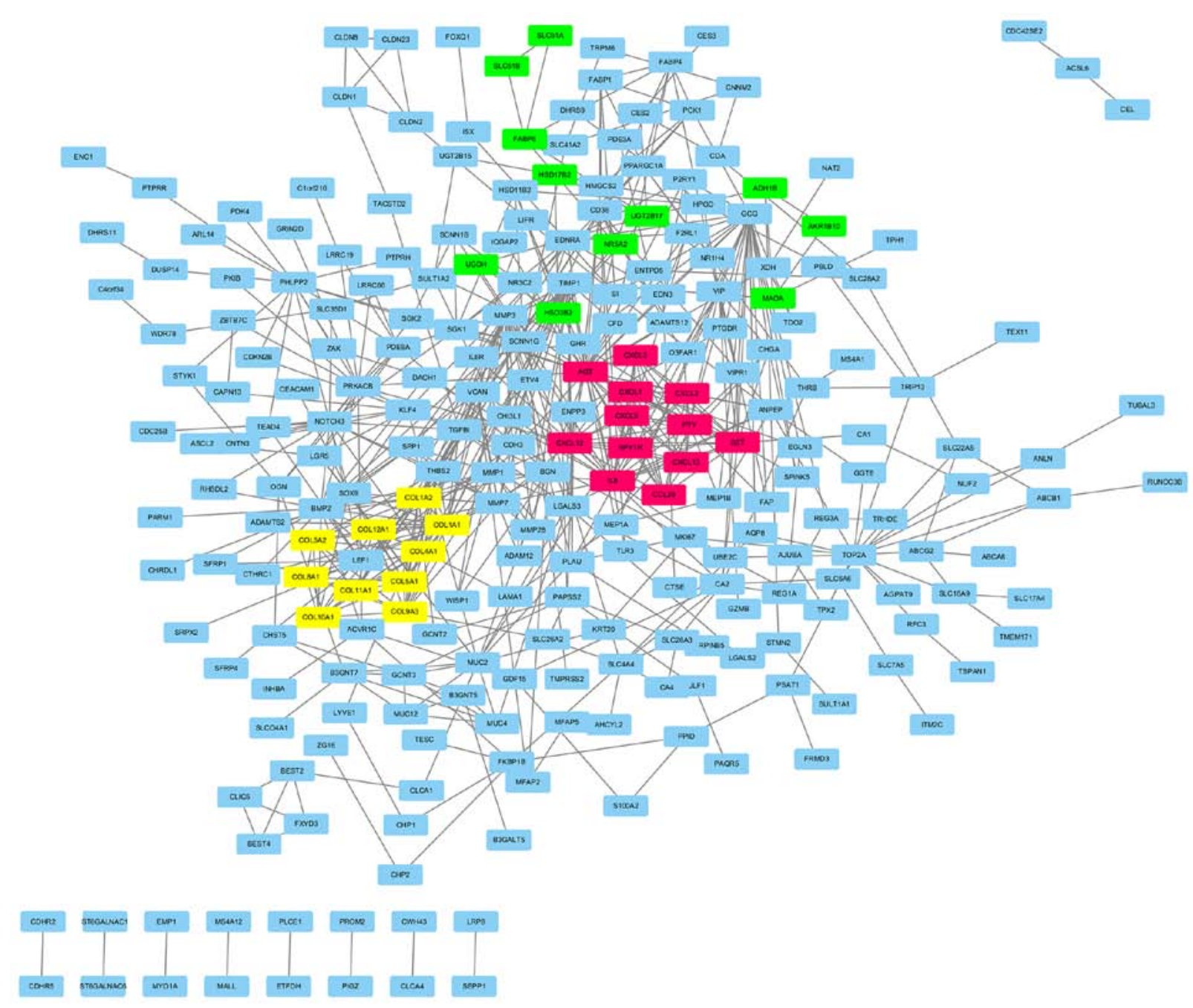

Figure 3. Protein-protein intersection (PPI) network of DEGs. All DEGs were uploaded to the Search Tool for the Retrieval of Interacting Genes (STRING) database to conduct PPI network for further study. The network was made up of 624 edges and 401 nodes with a combined score of $>0.4$. The red, yellow, green parts represent the top 3 modules of the PPI network.

The network consisted of 624 edges and 401 nodes (Fig. 3). To facilitate our understanding of DEGs, we visualized the network in the Cytoscape software and modularized it using plug-in MCODE. The results showed that these modules were mainly correlated with the chemokine signaling pathway, ECM-receptor interaction and androgen and estrogen metabolism (Fig. 4).

Moreover, the top 10 hub nodes with higher degrees were screened out, which included the upregulated genes that secreted protein acidic and rich in cysteine (SPARC), C-X-C motif chemokine ligand 3 (CXCL3), collagen, type IX, $\alpha 3$ (COL9A3), fatty acid binding protein 1 (FABP1), claudin-2 (CLDN2), and downregulated genes carbonic anhydrase IV (CA4), chromogranin A (CHGA), aldo-keto reductase family 1, member B10 (AKR1B10), transient receptor potential cation channel, subfamily M, member 6 (TRPM6) and FXYD domain containing ion transport regulator 3 (FXYD3) (Table II).

Comparison of expression data of the hub genes. To guarantee that the selected hub genes were credible, we analyzed their expression levels between colon cancer and normal tissues in TCGA and Oncomine. Expression levels of
Table II. Key differentially expressed genes (DEGs) obtained from the GSE39582 dataset.

\begin{tabular}{lcc}
\hline Gene symbol & LogFC & P-value \\
\hline SPARC & 2.00 & $9.76 \times 10^{-19}$ \\
CA4 & -5.45 & $2.81 \times 10^{-40}$ \\
CXCL3 & 2.36 & $3.51 \times 10^{-13}$ \\
COL9A3 & 2.06 & $1.54 \times 10^{-11}$ \\
FABP1 & 2.21 & $7.88 \times 10^{-12}$ \\
CHGA & -2.34 & $7.96 \times 10^{-29}$ \\
CLDN2 & 2.29 & $9.07 \times 10^{-10}$ \\
AKR1B10 & -4.44 & $3.29 \times 10^{-24}$ \\
TRPM6 & -1.50 & $3.02 \times 10^{-10}$ \\
FXYD3 & -2.21 & $6.78 \times 10^{-22}$ \\
\hline
\end{tabular}

The DEGs were identified between 566 colon cancer samples and 19 normal colon mucosae using Gene Expression Omnibus (GEO) 2R. The 10 hub genes were exported from the protein-protein intersection (PPI) network. 
A

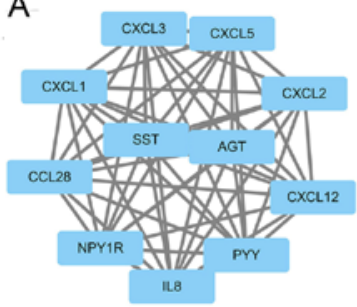

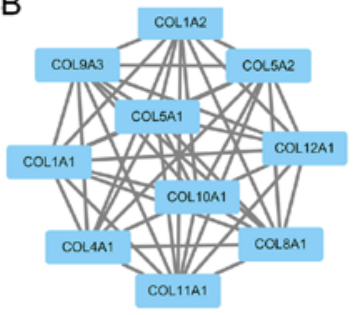

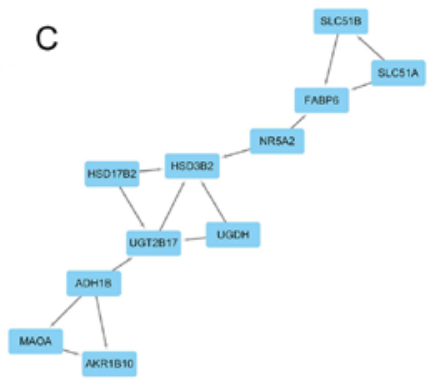

D

\begin{tabular}{|c|c|c|c|c|c|}
\hline Pathway ID & Name & $\begin{array}{l}\text { Gene } \\
\text { count }\end{array}$ & $\%$ & P-value & Genes \\
\hline hsa04062 & Chemokine signaling pathway & 7 & 63.6 & $6.02 \times 10^{-8}$ & $\begin{array}{l}\text { CXCL1, IL8, CXCL5, CXCL3, CXCL2, } \\
\text { CCL28, CXCL12 }\end{array}$ \\
\hline hsa04060 & Cytokine-cytokine receptor interaction & 7 & 63.6 & $4.54 \times 10^{-7}$ & $\begin{array}{l}\text { CXCL1, IL8, CXCL5, CXCL3, CXCL2, } \\
\text { CCL28, CXCL12 }\end{array}$ \\
\hline hsa04621 & NOD-like receptor signaling pathway & 3 & 27.3 & $3.91 \times 10^{-3}$ & CXCL1, IL8, CXCL2 \\
\hline \multicolumn{6}{|l|}{$E$} \\
\hline Pathway ID & Name & $\begin{array}{l}\text { Gene } \\
\text { count }\end{array}$ & $\%$ & P-value & Genes \\
\hline hsa04512 & ECM-receptor interaction & 6 & 60.0 & $1.09 \times 10^{-9}$ & $\begin{array}{l}\text { COL4A1, COL1A2, COL1A1, COL5A2, } \\
\text { COL11A1, COL5A1 }\end{array}$ \\
\hline hsa04510 & Focal adhesion & 6 & 60.0 & $9.20 \times 10^{-8}$ & $\begin{array}{l}\text { COL4A1, COL1A2, COL1A1, COL5A2, } \\
\text { COL11A1, COL5A1 }\end{array}$ \\
\hline \multicolumn{6}{|l|}{$\mathrm{F}$} \\
\hline Pathway ID & Name & $\begin{array}{l}\text { Gene } \\
\text { count }\end{array}$ & $\%$ & P-value & Genes \\
\hline hsa00150 & Androgen and estrogen metabolism & 3 & 33.3 & $1.40 \times 10^{3}$ & HSD3B2, UGT2B17, HSD17B2 \\
\hline hsa00140 & Steroid hormone biosynthesis & 3 & 33.3 & $2.17 \times 10^{-3}$ & HSD3B2, UGT2B17, HSD17B2 \\
\hline hsa00982 & Drug metabolism & 3 & 33.3 & $3.90 \times 10^{-3}$ & UGT2B17, MAOA, ADH1B \\
\hline hsa00053 & Ascorbate and aldarate metabolism & 2 & 22.2 & $2.65 \times 10^{-2}$ & UGT2B17, UGDH \\
\hline hsa00040 & Pentose and glucuronate interconversions & & 22.2 & $2.80 \times 10^{-2}$ & UGT2B17, UGDH \\
\hline
\end{tabular}

Figure 4. The top 3 modules of the PPI network. To screen out the hub genes from the modules, the PPI network was visualized in Cytoscape, and modules were established using the plug-in Molecular Complex Detection (MCODE) (http://apps.cytoscape.org/apps/mcode) with the MCODE scores of $>2$ and number of nodes $>9$ criteria. The top 3 modules, (A) module 1, (B) module 2, and (C) module 3, and its genes were exported in excel format. Additionally, DAVID was utilized to conduct the KEGG enrichment analysis of the genes within the modules separately. The results contained the enriched pathways of module (D) 1 , (E) 2 and (F) 3 .
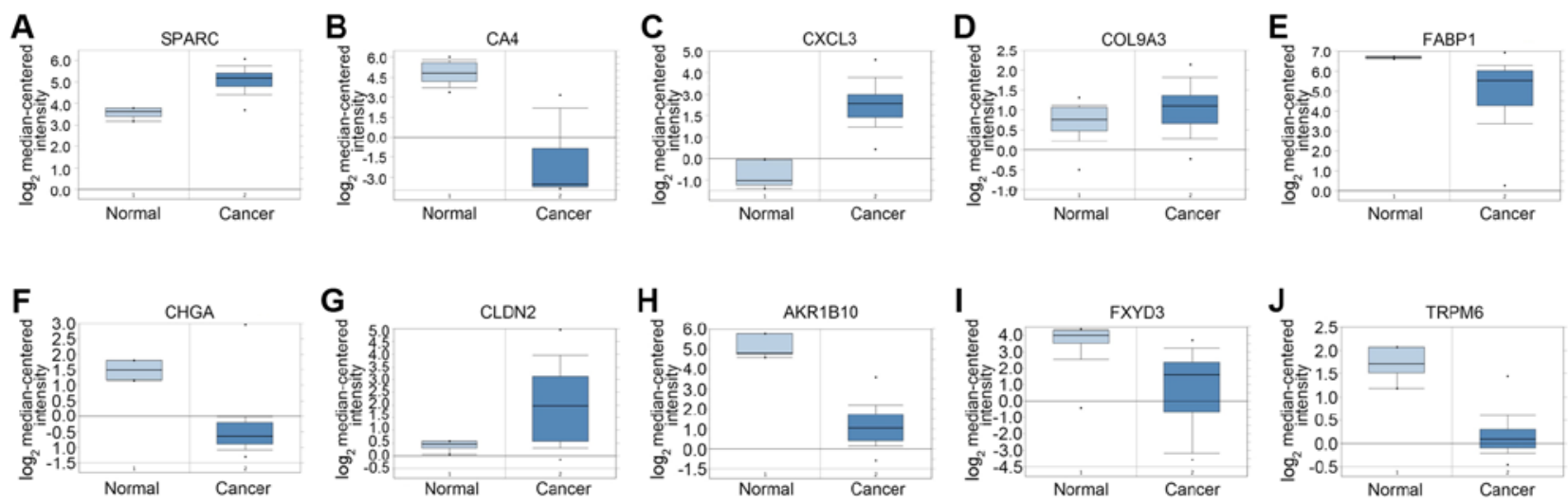

Figure 5. The expression of 10 hub genes in Oncomine database between colorectal adenocarcinoma and normal colon tissue. In order to verify the reliability of hub genes, such as (A) secreted protein acidic and rich in cysteine (SPARC), (B) carbonic anhydrase IV (CA4), (C) C-X-C motif chemokine ligand 3 (CXCL3), (D) collagen, type IX, $\alpha 3$ (COL9A3), (E) fatty acid binding protein 1 (FABP1), (F) chromogranin A (CHGA), (G) claudin-2 (CLDN2), (H) aldo-keto reductase family 1, member B10 (AKR1B10), (I) transient receptor potential cation channel, subfamily M, member 6 (TRPM6) and (J) FXYD domain containing ion transport regulator 3 (FXYD3), the expression of these genes was searched in Oncomine with the filter of Gene, Cancer vs. Normal Analysis and Colorectal cancer type. The results showed that all genes, except FABP1, had the same expression difference in the two groups with $\mathrm{P}<0.05$.

all the genes but FABP1 were consistent in these 3 different databases (Fig. 5 and Table III).

Analysis of the hub genes. Next, we further investigated the prognostic value of these genes. Survival curve was calculated according to the prognostic data of the patients. High expression of TRPM6 demonstrated a higher overall survival (OS) rate (Fig. 6A). In addition, the remaining hub genes were independent of OS (Fig. 6B-K). In this way, we exclusively focused on the abnormality of TRPM6, which was slightly 
A
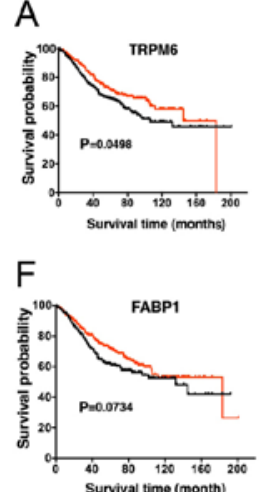

B

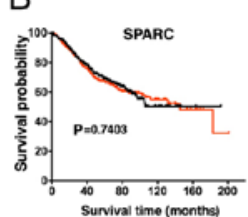

G

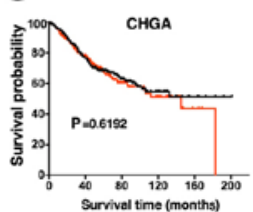

C

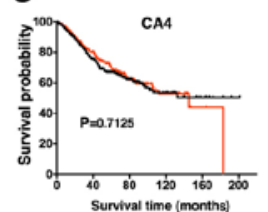

$\mathrm{H}$

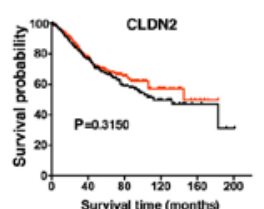

D

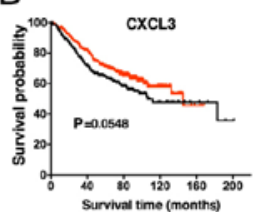

I

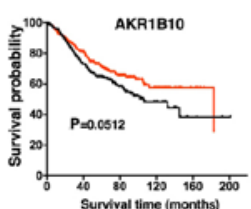

E

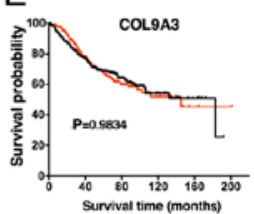

$J$

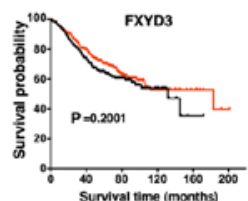

Figure 6. The prognosis analysis of 10 hub genes. Survival curves of hub genes were performed in GraphPad Prism software using prognosis information of GSE39582. The red and black curves represent the high and low expression groups, respectively. (A) The high expression of TRPM6 signified a better overall survival (OS) with P<0.05. In contast, (B) SPARC, (C) CA4, (D) CXCL3, (E) COL9A3, (F) FABP1, G) CHGA, (H) CLDN2, (I) AKR1B10 and (J) FXYD3 were not correlated with patient prognosis.

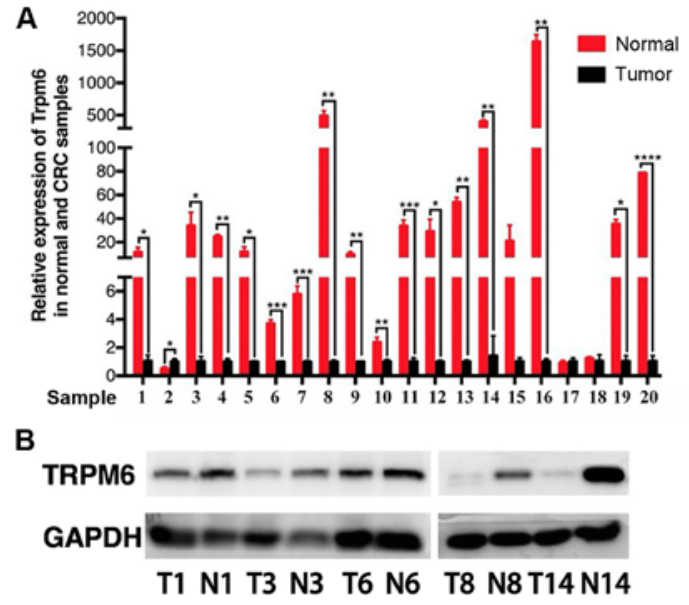

Figure 7. TRPM6 expression is downregulated in CRC. Experimental validation of TRPM6 in 20 paired clinical CRC samples. Compared with normal colon, (A) TRPM6 was downregulated in 16 of 20 paired human colon cancer samples by qPCR, (B) TRPM6 was downregulated in 6 paired human colon cancer samples by western blotting. CRC, colorectal cancer. T, tumor sample, N, normal sample. The results in the graph A were calculated using the Student's t-test. ${ }^{*} \mathrm{P}<0.05,{ }^{* *} \mathrm{P}<0.01,{ }^{* * * *} \mathrm{P}<0.001,{ }^{* * * * *} \mathrm{P}<0.0001$.

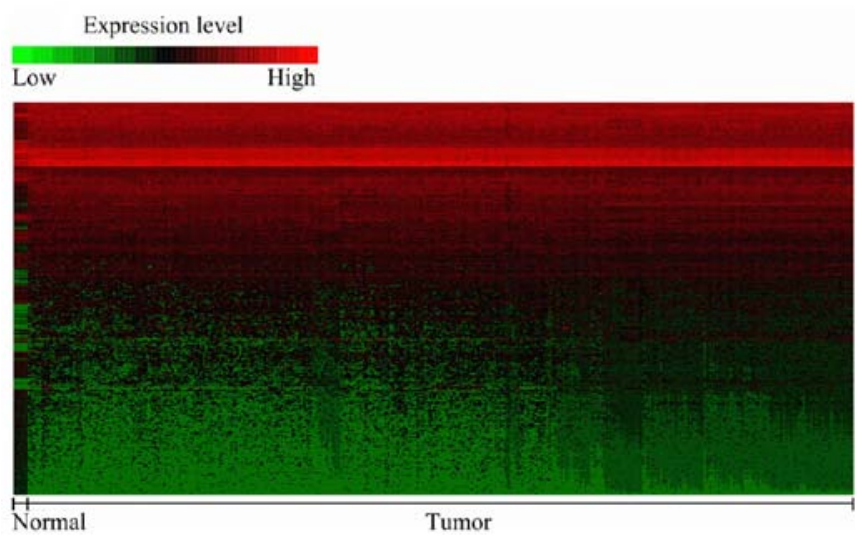

Figure 8. Heatmap of the differentially expressed microRNAs (DEMs) between colorectal adenocarcinoma and normal colon mucosa from TCGA A total of 516 DEMs were identified using edgeR package with $\mathrm{P}<0.05$ and $\log \mathrm{FC} \geq 1.0$. Then, heat map was performed using gplots package (https:// cran.r-project.org/web/packages/gplots/index.html). Coloring illustrates the high expression (red) and low expression (green) of the microRNAs.

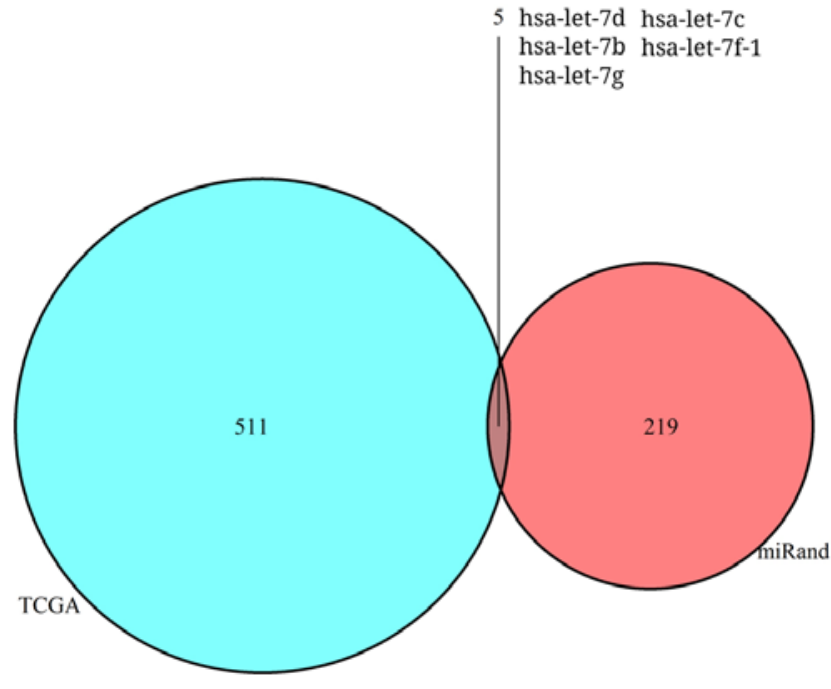

Figure 9. The regulatory microRNAs (miRNAs) of TRPM6. miRanda was utilized to predict the TRPM6-associated miRNAs with Human Good mirSVR score, Conserved miRNA and Good mirSVR score, and Non-conserved miRNA. Five miRNAs were exported after the intersection of the DEMs and miRanda results. The Venn diagram was constructed using VennDiagram package.

reported previously in CRC. We verified its expression level in clinical specimens by conducting qPCR and western blotting experiments (Fig. 7).

Hub gene TRPM6 maybe targeted by hsa-let-7g and hsa-let-7f-1. Owing to the significance of miRNAs in various diseases and their function in suppressing the translation of mRNAs, we analyzed DEMs between colon cancer and normal colon mucosa in TCGA. Compared with normal colon mucosa, 516 DEMs were identified in the colon adenocarcinoma group (Fig. 8). In addition, miRanda database was used to predict the regulatory miRNAs of TRPM6. The results showed that a total of 5 miRNAs were related with TRPM6 through the intersection of the results of TCGA and miRanda (Fig. 9 and Table IV). TRPM6 was downregulated in colon cancer, and hsa-let-7g and hsa-let-7f-1, which were upregulated in colon cancer, were predicted to be the key regulatory miRNAs of TRPM6. 
Table III. Expression of hub genes in DEGs from the TCGA database.

\begin{tabular}{lcc}
\hline Gene symbol & LogFC & P-value \\
\hline SPARC & 1.47 & $6.24 \times 10^{-14}$ \\
CA4 & -5.12 & $1.39 \times 10^{-80}$ \\
CXCL3 & 3.01 & $5.42 \times 10^{-28}$ \\
COL9A3 & 3.18 & $2.57 \times 10^{-15}$ \\
FABP1 & -2.78 & $1.73 \times 10^{-33}$ \\
CHGA & -4.54 & $2.44 \times 10^{-60}$ \\
CLDN2 & 5.49 & $2.50 \times 10^{-30}$ \\
AKRIB10 & -3.04 & $9.81 \times 10^{-44}$ \\
TRPM6 & -4.26 & $2.00 \times 10^{-122}$ \\
FXYD3 & -1.45 & $1.33 \times 10^{-22}$ \\
\hline
\end{tabular}

The differentially expressed genes (DEGs) were screened between 480 colorectal adenocarcinoma and 41 normal samples from The Cancer Genome Atlas (TCGA) using edgeR package. Expression of hub genes in Table I was verified in DEGs of the TCGA.

Table IV. Regulatory microRNAs (miRNAs) of TRPM6 in colon cancer.

\begin{tabular}{lcc}
\hline miRNA & LogFC & P-value \\
\hline hsa-let-7d & -2.81 & $1.70 \times 10^{-5}$ \\
hsa-let-7b & -2.66 & $3.90 \times 10^{-58}$ \\
hsa-let-7g & 2.07 & $1.41 \times 10^{-17}$ \\
hsa-let-7f-1 & 4.45 & $1.31 \times 10^{-10}$ \\
hsa-let-7c & -1.40 & $1.08 \times 10^{-3}$ \\
\hline
\end{tabular}

The differentially expressed miRNAs (DEMs) were acquired by comparing the expression of 457 colon adenocarcinoma samples with 8 normal colon samples from TCGA. miRanda database was used to predict the regulatory miRNAs of TRPM6. The results were obtained by the intersection between DEMs and miRanda from TCGA.

\section{Discussion}

In the present study, we explored the vital genes and their miRNAs in the development of CRC, which could enhance our insight of potential molecular mechanisms and benefit diagnosis, treatment and prognosis of the disease. In order to search the tumorigenesis-associated DEGs, we analyzed the gene expression profiling of GSE39582 with GEO2R and further identified 10 hub genes in 439 DEGs by Cytoscape software, which was tested by TCGA and Oncomine database. In terms of the prognostic value of TRPM6, qPCR was conducted in 20 paired colon mucosae samples to validate its role in diagnosis and prognosis of the disease. Moreover, the regulatory miRNAs of TRPM6 were predicted by TCGA and miRanda database. Combined with GO, KEGG and module analysis, our results showed that TRPM6 and other hub genes may play a critical role in the development of CRC. In order to further understand CRC, we innovatively and exclusively focused on the 10 hub genes and two miRNAs, hsa-let-7g and hsa-let-7f-1.

SPARC, CA4, CXCL3, FABP1, CLDN2, AKR1B10 and $F X Y D 3$ have previously been reported as genes that are tightly involved in the development of CRC (19-26). COL9A3, CHGA, and TRPM6 have been rarely studied and reported, but which may provide novel insight into the research of CRC. COL9A3 expression was intensely correlated with the expression of SOX10, a sensitive diagnostic marker for both salivary adenoid cystic carcinoma and basal-like breast carcinoma (27). CHGA, a biomarker in neuroendocrine tumors (NETs) (28), was downregulated in colon cancer tissues in this study. The accurate roles of these two genes, $C O L 9 A 3$ and $C H G A$, remain unclear in CRC.

TRPM6, a gene that belongs to the Transient receptor potential melastatin (TRPM) subfamily, is involved in the physiology of $\mathrm{Mg}^{2+}$ handling, which we exclusively focused on in our study. It was reported that TRPM6 mutations are associated with hypomagnesemia and downregulation of TRPM6 could result in hypomagnesemia (29). Magnesium plays a role in modulating cellular biochemical reactions, such as differentiation, proliferation, apoptosis and migration (30). Many studies have confirmed that hypomagnesemia could promote tumor metastasis $(31,32)$. To date, few reports have discussed the role of TRPM6 in tumors. We found that the upregulation of TRPM6 was associated with prolonged OS in patients, although the survival analysis $(\mathrm{P}=0.0498)$ was marginally significant. Considering the limited sample sizes and that only TRPM6 was statistically significant under the same grouping condition, we hypothesized that the level of TRPM6 expression was related to the survival and prognosis of colon cancer patients. Then we tested the mRNA expression level of TRPM6 in patient samples using qPCR. The results revealed that $80 \%$ samples showed decreased expression of TRPM6 in colon cancer samples compared with their paired normal tissues. Therefore, we considered TRPM6 as a promising biomarker of tumorigenesis and a treatment target in CRC patients, although the mechanisms of TRPM6 in CRC are still not completely clear. We did not examine expression of all hub genes in the colon cancer samples by qPCR and western blotting and this was a limitation of the study. We assumed that the expression trends of the other hub genes should be in accordance with the results (Tables II and III) based on previous analysis and reports from other investigators (19-26), which need to be experimentally verified in further research.

Hsa-let-7g, a member of the let-7 family playing a vital role in tumorigenesis, was demonstrated to dramatically inhibit the proliferation of hepatocellular carcinoma by downregulating the expression of the oncogene, c-Myc, and upregulating the expression of anti-oncogene, p16(INK4A) (33). Moreover, hsa-let-7g was found to participate in the process of the regulation of autophagy and apoptosis by modulating LOX-1 in vascular smooth muscle cells (34), and an apoptosis-promoting function was also observed in gastric cancer. The relationship between hsa-let-7g and TRPM6 was not validated, but its high-expression trend in colon cancer increased the possibility of the prediction. Similarly, hsa-let-7f-1 is also a member of let-7 family, but research concerning hsa-let-7f-is limited. According to a previous study (35), hsa-let-7f-1 is involved in 
the Notch signaling pathway, which is closely associated with the development of CRC $(36,37)$. The role of hsa-let-7f-1 and the evidence of the regulatory relationship between hsa-let-7f-1 and TRPM6 should be further elucidated.

In summary, we identified 439 DEGs and 516 DEMs using GEO and the TCGA database between colon cancer and normal colon mucosa. Many of these DEGs, such as SPARC, CA4, CXCL3, FABP1, CLDN2, AKR1B10, FXYD3, COL9A3, CHGA, TRPM6, hsa-let-7g and hsa-let-7f-1, were predicted to be vital molecules related to $\mathrm{CRC}$ tumorigenesis. We also analyzed the GSE39582 dataset and found the DEGs of right vs. left colon cancer were not relevant with our hub genes in this study (data not shown). There are also some limitations to our study. For instance, 20 pairs of samples were still not enough and we still have to further verify the expression level of TRPM6 using more samples. We also did not detect the mutations of TRPM6 and confirm the relationship between the mutations and the decreased expression of TRPM6 in colon cancer. In general, our research provides a series of promising targets for diagnosis, treatment and prognosis and provides insight for further investigation into the potential underlying mechanisms.

\section{Acknowledgements}

The study represents partial fulfillment of the requirements for a Doctor degree for Dr Binbin Xie.

\section{Funding}

The present study was supported by the National Natural Science Foundation of China (grant nos. 81572592, 81772543, 81572361), the Zhejiang Natural Sciences Foundation Grant (LY15H160025, LQ16H160003), the Zhejiang Province Preeminence Youth Fund (LR16H160001), the Zhejiang Medical Innovative Discipline Construction Project-2016, and the Hangzhou Health and Family Planning Commission Fund (2014A61).

\section{Availability of data and materials}

The datasets analyzed during the current study are available from the corresponding author on reasonable request.

\section{Authors' contributions}

$\mathrm{BX}, \mathrm{RZ}$ and $\mathrm{BB}$ designed the study and drafted the manuscript. BX, YW, YX and SL carried out the experiments. XZ, $\mathrm{ZW}$ and YF participated in the statistical analysis of the data. EPM, WH and HP are responsible for the revision of the manuscript and were also involved in the conception of the study. All authors read and approved the manuscript and agree to be accountable for all aspects of the research in ensuring that the accuracy or integrity of any part of the work are appropriately investigated and resolved.

\section{Ethics approval and consent to participate}

Research was authorized by the Ethics Committee of Sir Run Run Shaw Hospital and informed consent was obtained from all participating patients. The reference no. was 20180226-88.

\section{Patient consent for publication}

Not applicable.

\section{Competing interests}

The authors declare that they have no competing interests.

\section{References}

1. Arnold M, Sierra MS, Laversanne M, Soerjomataram I, Jemal A and Bray F: Global patterns and trends in colorectal cancer incidence and mortality. Gut 66: 683-691, 2017.

2. Siegel RL, Miller KD, Fedewa SA, Ahnen DJ, Meester RGS, Barzi A and Jemal A: Colorectal cancer statistics, 2017. CA Cancer J Clin 67: 177-193, 2017.

3. Aran V, Victorino AP, Thuler LC and Ferreira CG: Colorectal cancer: Epidemiology, disease mechanisms and interventions to reduce onset and mortality. Clin Colorectal Cancer 15: 195-203, 2016.

4. Hollstein M, Sidransky D, Vogelstein B and Harris CC: p53 mutations in human cancers. Science 253: 49-53, 1991.

5. Herman JG, Umar A, Polyak K, Graff JR, Ahuja N, Issa JP, Markowitz S, Willson JK, Hamilton SR, Kinzler KW, et al: Incidence and functional consequences of $h M L H 1$ promoter hypermethylation in colorectal carcinoma. Proc Natl Acad Sci USA 95: 6870-6875, 1998.

6. Reya T and Clevers H: Wnt signalling in stem cells and cancer. Nature 434: 843-850, 2005.

7. Brenner H, Kloor M and Pox CP: Colorectal cancer. Lancet 383: 1490-1502, 2014

8. Wierzbicki PM and Rybarczyk A: The Hippo pathway in colorectal cancer. Folia Histochem Cytobiol 53: 105-119, 2015.

9. Papadatos-Pastos D, Rabbie R, Ross P and Sarker D: The role of the PI3K pathway in colorectal cancer. Crit Rev Oncol Hematol 94: 18-30, 2015.

10. Anastas JN and Moon RT: WNT signalling pathways as therapeutic targets in cancer. Nat Rev Cancer 13: 11-26, 2013.

11. Asangani IA, Rasheed SA, Nikolova DA, Leupold JH, Colburn NH, Post S and Allgayer H: MicroRNA-21 (miR-21) post-transcriptionally downregulates tumor suppressor Pdcd4 and stimulates invasion, intravasation and metastasis in colorectal cancer. Oncogene 27: 2128-2136, 2008.

12. Iwaya T, Yokobori T, Nishida N, Kogo R, Sudo T, Tanaka F, Shibata K, Sawada G, Takahashi Y, Ishibashi M, et al: Downregulation of $m i R-144$ is associated with colorectal cancer progression via activation of $m T O R$ signaling pathway. Carcinogenesis 33: 2391-2397, 2012.

13. Yan M, Song M, Bai R, Cheng S and Yan W: Identification of potential therapeutic targets for colorectal cancer by bioinformatics analysis. Oncol Lett 12: 5092-5098, 2016.

14. Marisa L, de Reyniès A, Duval A, Selves J, Gaub MP, Vescovo L, Etienne-Grimaldi MC, Schiappa R, Guenot D, Ayadi M, et al: Gene expression classification of colon cancer into molecular subtypes: Characterization, validation, and prognostic value. PLoS Med 10: e1001453, 2013.

15. Barrett T, Wilhite SE, Ledoux P, Evangelista C, Kim IF, Tomashevsky M, Marshall KA, Phillippy KH, Sherman PM, Holko M, et al: NCBI GEO: Archive for functional genomics data sets-update. Nucleic Acids Res 41: D991-D995, 2013.

16. Gaudet P, Skunca N, Hu JC and Dessimoz C: Primer on the gene ontology. Methods Mol Biol 1446: 25-37, 2017.

17. Kanehisa M, Furumichi M, Tanabe M, Sato Y and Morishima K: KEGG: New perspectives on genomes, pathways, diseases and drugs. Nucleic Acids Res 45: D353-D361, 2017.

18. Livak KJ and Schmittgen TD: Analysis of relative gene expression data using real-time quantitative PCR and the $2^{-\Delta \Delta C_{\mathrm{T}}}$ Method. Methods 25: 402-408, 2001.

19. Takemasa I, Higuchi H, Yamamoto H, Sekimoto M, Tomita N, Nakamori S, Matoba R, Monden $M$ and Matsubara K: Construction of preferential cDNA microarray specialized for human colorectal carcinoma: Molecular sketch of colorectal cancer. Biochem Biophys Res Commun 285: 1244-1249, 2001.

20. Zhang J, Tsoi H, Li X, Wang H, Gao J, Wang K, Go MY, Ng SC, Chan FK, Sung JJ, et al: Carbonic anhydrase IV inhibits colon cancer development by inhibiting the Wnt signalling pathway through targeting the WTAP-WT1-TBL1 axis. Gut 65: 1482-1493, 2016. 
21. Farquharson AJ, Steele RJ, Carey FA and Drew JE: Novel multiplex method to assess insulin, leptin and adiponectin regulation of inflammatory cytokines associated with colon cancer. Mol Biol Rep 39: 5727-5736, 2012.

22. Wood SM, Gill AJ, Brodsky AS, Lu S, Friedman K, Karashchuk G, Lombardo K, Yang D and Resnick MB: Fatty acid-binding protein 1 is preferentially lost in microsatellite instable colorectal carcinomas and is immune modulated via the interferon $\gamma$ pathway. Mod Pathol 30: 123-133, 2017.

23. Gaspar $\mathrm{C}$ and Fodde $\mathrm{R}: A P C$ dosage effects in tumorigenesis and stem cell differentiation. Int J Dev Biol 48: 377-386, 2004.

24. Wu P, Wu D, Ni C, Ye J, Chen W, Hu G, Wang Z, Wang C, Zhang Z, Xia W, et al: $\gamma \delta \mathrm{T} 17$ cells promote the accumulation and expansion of myeloid-derived suppressor cells in human colorectal cancer. Immunity 40: 785-800, 2014.

25. Zu X, Yan R, Pan J, Zhong L, Cao Y, Ma J, Cai C, Huang D, Liu J, Chung FL, et al: Aldo-keto reductase 1B10 protects human colon cells from DNA damage induced by electrophilic carbonyl compounds. Mol Carcinog 56: 118-129, 2017.

26. Widegren E, Onnesjö S, Arbman G, Kayed H, Zentgraf H, Kleeff J, Zhang H and Sun XF: Expression of FXYD3 protein in relation to biological and clinicopathological variables in colorectal cancers. Chemotherapy 55: 407-413, 2009.

27. Ivanov SV, Panaccione A, Nonaka D, Prasad ML, Boyd KL, Brown B, Guo Y, Sewell A and Yarbrough WG: Diagnostic SOX10 gene signatures in salivary adenoid cystic and breast basal-like carcinomas. Br J Cancer 109: 444-451, 2013.

28. Krampitz GW, George BM, Willingham SB, Volkmer JP, Weiskopf K, Jahchan N, Newman AM, Sahoo D, Zemek AJ, Yanovsky RL, et al: Identification of tumorigenic cells and therapeutic targets in pancreatic neuroendocrine tumors. Proc Natl Acad Sci USA 113: 4464-4469, 2016.
29. Vincenzi B, Santini D and Tonini G: Biological interaction between anti-epidermal growth factor receptor agent cetuximab and magnesium. Expert Opin Pharmacother 9: 1267-1269, 2008.

30. Wolf FI and Trapani V: Cell (patho)physiology of magnesium. Clin Sci 114: 27-35, 2008.

31. Nasulewicz A, Wietrzyk J, Wolf FI, Dzimira S, Madej J, Maier JA, Rayssiguier Y, Mazur A and Opolski A: Magnesium deficiency inhibits primary tumor growth but favors metastasis in mice. Biochim Biophys Acta 1739: 26-32, 2004.

32. Solinas G, Marchesi F, Garlanda C, Mantovani A and Allavena P: Inflammation-mediated promotion of invasion and metastasis. Cancer Metastasis Rev 29: 243-248, 2010.

33. Lan FF, Wang H, Chen YC, Chan CY, Ng SS, Li K, Xie D, He ML, Lin MC and Kung HF: Hsa-let-7g inhibits proliferation of hepatocellular carcinoma cells by downregulation of $c-M y c$ and upregulation of $p 16^{I N K 4 A}$. Int J Cancer 128: 319-331, 2011.

34. Ding Z, Wang X, Schnackenberg L, Khaidakov M, Liu S, Singla S, Dai Y and Mehta JL: Regulation of autophagy and apoptosis in response to ox-LDL in vascular smooth muscle cells, and the modulatory effects of the microRNA hsa-let-7 g. Int J Cardiol 168: 1378-1385, 2013.

35. Huang S, Feng C, Zhai YZ, Zhou X, Li B, Wang LL, Chen W, Lv FQ and Li TS: Identification of miRNA biomarkers of pneumonia using RNA-sequencing and bioinformatics analysis. Exp Ther Med 13: 1235-1244, 2017.

36. Wang Y, Huang D, Chen KY, Cui M, Wang W, Huang X, Awadellah A, Li Q, Friedman A, Xin WW, et al: Fucosylation deficiency in mice leads to colitis and adenocarcinoma. Gastroenterology 152: 193-205.e10, 2017.

37. Srinivasan T, Walters J, Bu P, Than EB, Tung KL, Chen KY, Panarelli N, Milsom J, Augenlicht L, Lipkin SM, et al: NOTCH signaling regulates asymmetric cell fate of fast- and slow-cycling colon cancer initiating cells. Cancer Res 76: 3411-3421, 2016. 